\title{
Flight laser module monitoring during ExoMars Raman laser spectrometer environmental testing
}

Pablo Rodriguez, Marina Benito, César Quintana, Carlos Pérez, Andoni Moral

Pablo Rodriguez, Marina Benito, César Quintana, Carlos Pérez, Andoni Moral, "Flight laser module monitoring during ExoMars Raman laser spectrometer environmental testing," Proc. SPIE 11852, International Conference on Space Optics - ICSO 2020, 118522R (11 June 2021); doi: $10.1117 / 12.2599446$

SPIE Event: International Conference on Space Optics - ICSO 2021, 2021, Online Only 


\section{International Conference on Space Optics-ICSO 2020}

Virtual Conference

30 March-2 April 2021

Edited by Bruno Cugny, Zoran Sodnik, and Nikos Karafolas
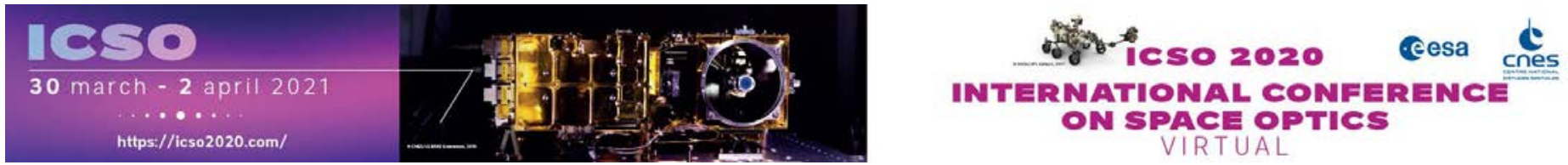

\section{Flight laser module monitoring during ExoMars Raman laser spectrometer environmental testing}

\section{Cesa isoporecestings denes}




\title{
Flight laser module monitoring during ExoMars Raman Laser Spectrometer environmental testing
}

\author{
Pablo Rodríguez*a ${ }^{*}$ Marina Benito ${ }^{\mathrm{b}}$, César Quintana ${ }^{\mathrm{a}}$, Carlos Pérez $^{\mathrm{a}}$, Andoni Moral $^{\mathrm{a}}$ \\ anstituto Nacional de Técnica Aeroespacial. Crta. Ajalvir, km 4. Torrejón de Ardoz (Spain) \\ bIngeniería de Sistemas para la Defensa de España. C/ Beatriz de Bobadilla, 3. Madrid (Spain)
}

\begin{abstract}
The flight units of the laser module for the Raman Laser Spectrometer (RLS) instrument of the ExoMars mission were manufactured, tested and finally delivered in March 2018. Based on the data collected during flight batch acceptance testing, FM and FS lasers were picked from that batch, and the former was subsequently tested at the next integration levels, i.e. RLS instrument, Analytical Laboratory Drawer (ALD) and ExoMars rover itself. As the system complexity increased, the data available for laser performance assessment became sparse due to shorter testing slots and to the basic information available through instrument telemetries and products. In order to assess the status of the laser module during these years of on-ground testing we have developed tools to extract as much information as possible from test sessions. Our starting inputs were some relevant house-keepings, such as laser optical power and temperature, and Raman spectra, from where laser spectral signature was obtained. Our analysis of the available on-ground test sessions at ALD and rover level show that the laser unit is working properly, but a fine tune of the working temperature setpoint may be required to provide optimal scientific return. The procedures developed will be also highly valuable for the definition of the laser performance assessment engineering tools during standard scientific operation in Mars.
\end{abstract}

Keywords: ExoMars, Raman spectrometer, laser, environmental tests

\section{INTRODUCTION}

\subsection{ExoMars 2022 and the Raman Laser Spectrometer}

The next ExoMars mission, an astrobiology program by ESA and the Russian space agency Roscosmos, will be launched in September 2022 and reach its destination in June 2023. The mission scientific goals comprise searching for biosignatures of past Martian life, characterizing water and geochemical distribution as a function of depth to better understand the evolution and habitability of Mars, and achieving incremental steps for future sample return missions. In order to achieve these objectives, the ExoMars rover will study the composition of samples extracted up to $2 \mathrm{~m}$ below the surface. These samples will be processed and analyzed by a suite of instruments in the so-called Analytical Laboratory Drawer (ALD). One of these three instruments is the Raman Laser Spectrometer (RLS), which seeks to search biosignatures and biomarkers on Mars using Raman spectroscopy ${ }^{1}$. By applying this technique we expect to identify mineral phases produced by water-related processes. In addition, the instrument could also identify organic compounds and aid in the search for microbial life by detecting several markers of biological activities. RLS will provide geological and mineralogical context information with a non-destructive technique that permits the analysis of the same sample by the other instruments in the ALD, and therefore richer, cross-correlated scientific data ${ }^{1}$.

The RLS instrument is a modular Raman spectrometer formed by three main units: the Spectrometer Unit (SPU), the Internal Optical Head (iOH) and the Instrument Control and Excitation Unit (ICEU). The Raman Laser Module (RLM) is located inside the ICEU, the instrument electronic box, and its excitation signal is carried by means of optical harness to the iOH to illuminate the sample. The optical head also collects the Raman signal and couples it to another fibre for transmission to the SPU, where the Raman spectrum is finally registered. A detailed description of the instrument can be found in the literature ${ }^{1}$.

*rodriguezpp@inta.es; www.inta.es 


\subsection{RLS Laser Module}

The RLS Laser Module (RLM) is a diode-pumped solid-state (DPSS) delivering a target 20-30mW optical power at $532.2 \mathrm{~nm}$ with a high wavelength stability $(+/-10 \mathrm{pm})$, narrow linewidth $(<30 \mathrm{pm})$ and high side-mode suppression ratio $(>20 \mathrm{~dB})$. The flight model includes two redundant resonators plus the necessary optics to co-align the beams and to couple them into the unit output optical fiber connector ${ }^{1}$. In addition, the laser has two redundant thermal sensors for thermal control and two internal photodiodes, to monitor the optical power and the returning signal from the sample, which is used to focus the instrument ${ }^{1}$. The development of the RLS laser module has been a big challenge, especially when it comes to the technology used to attach the optical components to their baseplate ${ }^{2}$. All this effort was paid off when the flight units were manufactured, tested and finally delivered back in March 2018, at a time when ExoMars rover launch date was still scheduled for summer 2020. The complete RLM AIV campaign was led by the National Institute for Aerospace Technology, INTA (Spain), and included three main subcontractors and more than fifteen different providers for just a $60 \mathrm{~g}$ mass unit. INTA was also responsible for some of the integration processes as well as of the complete test campaign. Key partners contributing to this successful delivery were Monocrom (Spain) and the Fraunhofer Institute of Jena (Germany) for the laser head, an AlN substrate with the micro-optical components properly aligned and fixed (soldered) on metallic pads. LIDAX (Spain) was responsible for the laser housing, laser head fixation on it, housing sealing and the overall thermo-mechanical design, while internal and external electrical connections were performed at INTA. Scientific partners in the RLS instrument guaranteed the final laser performance by providing its mechanical fixation and electrical control (Institut de Recherche en Astrophysique et Planétologie, IRAP, France) as well as the required tight thermal control (IRAP, France and Rutherford Appleton Laboratory, RAL, UK), achieved by means of a thermo-electric module (TEM) controlled by a PID algorithm running on the instrument FPGA.
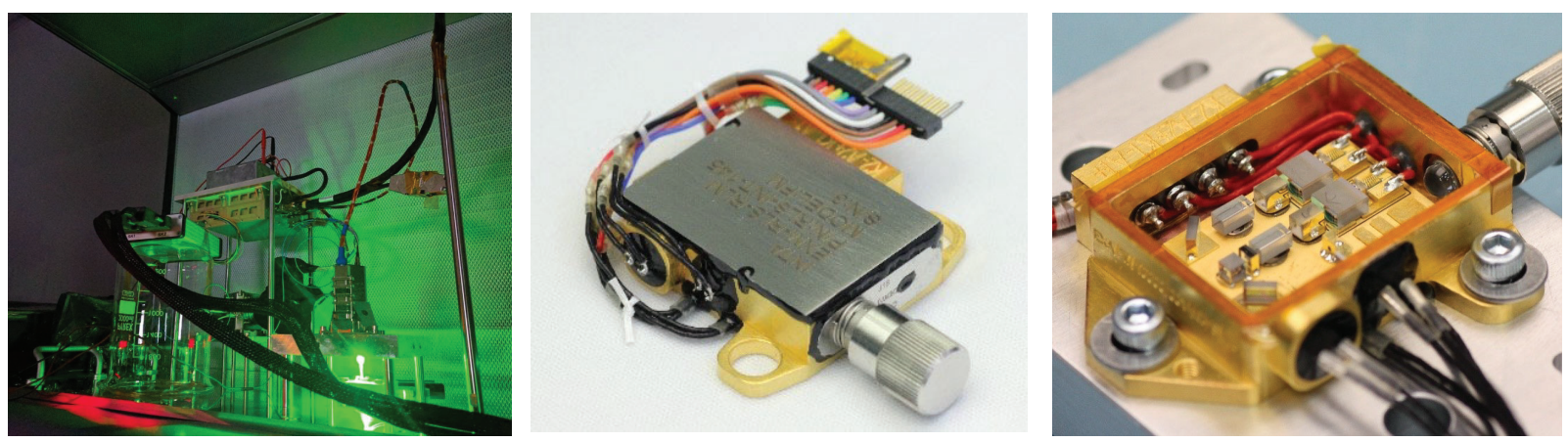

Figure 1. RLS instrument in ALD-like test configuration at INTA (left). Flight Model of Raman Laser Module as delivered (center) and during the assembly phase (right).

The environmental test sequence of the flight laser modules prior to its integration into the RLS instrument included a preliminary screening of the complete flight batch at INTA. The screening was based on a random vibration test at acceptance levels (7.86 grms during 60s, only in the out-of-plane, worst axis) plus two thermal cycles, again at acceptance levels $\left(-55^{\circ} \mathrm{C}\right.$ to $+65^{\circ} \mathrm{C}$ with a maximum slope of $\left.5^{\circ} \mathrm{C} / \mathrm{min}\right)$. From this screening, three flight candidates out of six units were identified and subsequently submitted to a full optical characterization ${ }^{3}$. This characterization included as main outcome a 2D map of delivered optical power and side-mode suppression ratio (SMSR) across the operational temperature range $\left(20^{\circ} \mathrm{C}\right.$ to $\left.30^{\circ} \mathrm{C}\right)$ and for a wide range of polarization currents, from lasing threshold $(0.5 \mathrm{~A})$ to a maximum of 2A. Flight unit selection was based on two criteria: robust, i.e. insensitive to current or temperature changes, SMSR and high slope efficiency. Even though the laser driver was conceived to work in constant output power mode at a single fixed output (closed loop controlled by a feedback photodiode); by tuning the laser temperature setpoint, operation could be changed to constant current at the minimum provided by the flight driver (0.9A). In these conditions, output power is slightly higher than originally required and both power stability and power repeatability are expected to fluctuate around $\pm 5 \%$, but this is highly compensated by the extended current range available for increasing output power during operation. In addition, constant current operation provides an even more stable environment inside the laser and an overall better spectral repeatability. 
Before integration into the instrument, the laser modules underwent a formal acceptance sequence inside RLS electronic box (Instrument Control and Excitation Unit, ICEU), including three-axis vibration, non-operational thermal cycles and operational thermal testing in vacuum conditions.

\subsection{Environmental testing sequence}

Once the RLS instrument was delivered to Thales Alenia Space (Torino, Italy), it was integrated into the ALD, tested, and then transferred to the ExoMars rover team in Airbus (Stevenage, UK). As the system complexity increased, the testing environment became more realistic but the data available for laser performance assessment were not so extensive due to shorter testing slots and to the basic information available through instrument telemetries and products. During its stand-alone testing, direct measurements of the laser beam were performed with fast, high precision power meters and high resolution $(6 \mathrm{pm})$ Fourier transform spectrometers, among other equipment. Once inside the RLS instrument, the data gathered were poorer, with just some relevant house-keepings updated at $0.1 \mathrm{~Hz}$, like temperature and the laser optical power as measured internally by the embedded photodiode. As for the spectral performance, it could be assessed indirectly from the RLS images by analyzing the laser signature on the sample Raman spectra and due to Rayleigh scattering, but with a much lower resolution (0.1-0.2nm) and with more sources of noise (dark current, Raman fluorescence, Raman linewidth...).

However sparse and limited, these data were quite interesting at least for two reasons: (a) the detection of any potential fluctuation in the RLS FM laser performance during the (two) years already expended in on-ground AIV after its formal delivery, and (b) the preparation of engineering tools for laser performance assessment during real operation, just based on the data provided by the instrument itself.

\section{METHODS}

\subsection{Data extraction and processing}

RLS test sessions at instrument and ALD level shared a common storage format, based on the use of the Rover Vehicle Interface Simulator (RVIS). Tools to analyze RVIS sessions had been developed in the context of the project ${ }^{4}$ and they were used to extract relevant data from them. At rover level, data were provided directly by the on-board computer (OBC). For batch processing, the rover sessions had to be pre-processed first and parsed to RVIS sessions.

Once all measurement sessions were in a common format, house-keeping (HK) data were exported to datasheets and Raman spectra to images. Among the examined HK were optical power, as monitored by the laser module internal photodiode, autofocus signal, also monitored by a laser internal photodiode, and laser housing temperature setpoint. Engineering data processing will be facilitated with the tools under preparation for operation on Mars ${ }^{5}$. As part of the preparation activities, the analysis of the ground testing sessions is already providing useful insights into the kind of complex analyses required for extracting as much information as possible from the instrument telemetries.

Raman spectra were processed in order to extract meaningful SMSR estimates. By default, the automatic exposure time algorithm inside RLS optimizes the dynamic range of the Raman spectrum. This led sometimes to saturated, unreliable, laser lines if Rayleigh scattering from the sample was high (or Raman signal low). Therefore laser line saturation status need to be checked in all captured images. Afterwards, background noise was cancelled, using dark images when available or estimating dark noise from the borders of the image. Most of the Raman spectra obtained during formal testing were from the RLS calibration target $^{6}$, a marked, well-characterized piece of polyethylene terephthalate (PET). This material creates a steep fluorescence background that was also removed before further processing. Finally, images were normalized and the SMSR was estimated at the PET main Raman peak (Raman shift, $1691 \mathrm{~cm}^{-1}$ ). Due to the proximity of the laser module main $(532.2 \mathrm{~nm})$ and secondary peak $(531.5 \mathrm{~nm})$, this main Raman line could be also adjusted to a Gaussian and suppressed so its contribution to the secondary peak was not taken into account. This procedure is relevant only for the Raman spectrum, where intrinsic linewidth may be problematic. 

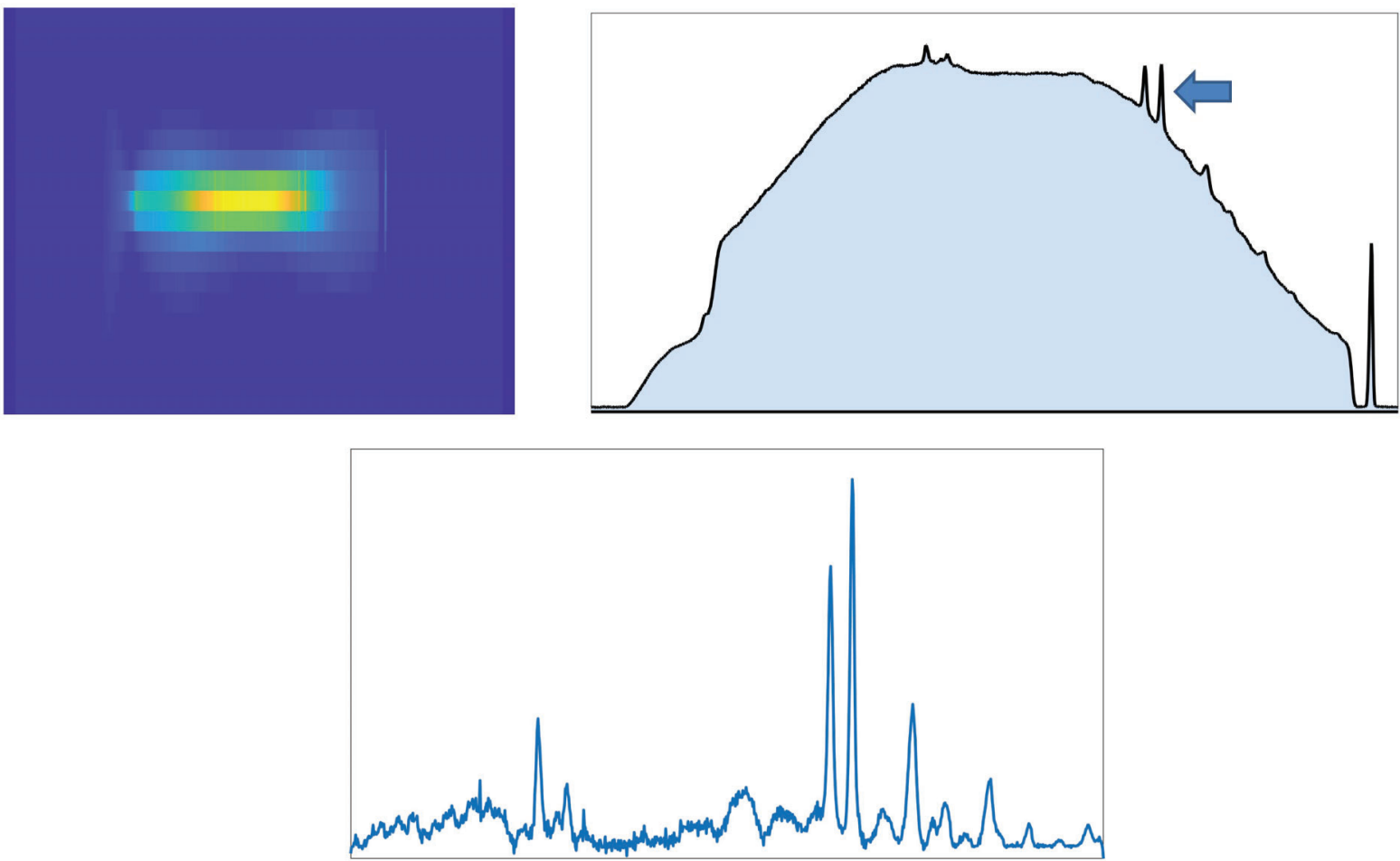

Figure 2. RLS calibration target spectrum, as provided by the SPU detector (upper panel, left) and binned (upper panel, right). Note the high contribution of fluorescence (the "hump"). The PET peak used for laser module spectral assessment, typically measured with the highest signal-to-noise ratio, is marked by the arrow. Removal of fluorescence helps to identify the sample Raman peaks (lower panel).

\subsection{Test sessions}

Next table contains the test sessions analyzed, listed chronologically. We focused on the more representative sessions: vacuum or low-pressure $\mathrm{CO}_{2}$ environments at several thermal conditions. Along this time, some quick, minor checks (before and after transportation, for example) have been performed, but they are not included here because in most cases a short, reduced functional, not including laser operation, was performed on the RLS instrument.

Table 1. Analyzed test sessions. Laser channel 1 in the RLM was switched on in all the sessions, sometimes twice (indicated by letters $a$ and $b$ in test ID). Raman spectra were not always taken. GTM stands for Ground Terrain Model.

\begin{tabular}{ccclc} 
Test ID & Level & Date & Description & Spectra (Y/N) \\
\hline 1 & ALD & $05 / 03 / 2019$ & Thermal test: $7 \mathrm{mbar}^{\mathrm{CO}} \mathrm{CO}_{2}, 22^{\circ} \mathrm{C}$ & $\mathrm{Y}$ \\
2a, $2 \mathrm{~b}$ & ALD & $08 / 03 / 2019$ & Thermal test: 7 mbar $\mathrm{CO}_{2},+10^{\circ} \mathrm{C}$ & $\mathrm{Y}$ \\
$3 \mathrm{a}, 3 \mathrm{~b}$ & ALD & $09 / 03 / 2019$ & Thermal test: 7 mbar $\mathrm{CO}_{2},-20^{\circ} \mathrm{C}$ & $\mathrm{Y}$ \\
4 & ALD & $13 / 03 / 2019$ & Thermal test: $7 \mathrm{mbar} \mathrm{CO}_{2},-40^{\circ} \mathrm{C}$ & $\mathrm{Y}$ \\
5 & ALD & $20 / 03 / 2019$ & Thermal test: $7 \mathrm{mbar} \mathrm{CO}_{2},+22^{\circ} \mathrm{C}$ & $\mathrm{Y}$ \\
6 & Rover & $22 / 08 / 2019$ & GTM test: 1 atm, $+22^{\circ} \mathrm{C}$ (room conditions) & $\mathrm{N}$ \\
7 & Rover & $25 / 10 / 2019$ & Post-acoustic test: 1 atm, $+22^{\circ} \mathrm{C}$ (room conditions) & $\mathrm{N}$ \\
8 & Rover & $12 / 11 / 2019$ & Thermal test: vacuum, $-40^{\circ} \mathrm{C}$ & $\mathrm{Y}$
\end{tabular}




\section{RESULTS}

\subsection{Thermal control}

Switching on and off the laser module creates the biggest perturbation for its thermal control system. We monitored this part of the house-keeping closely (see Figure 3) in order to detect changes in the settling time or temperature overshoot that could be correlated with a degradation in the thermal contact or with high currents required suddenly by the unit. The results show that settling pattern is consistent, with an average overshoot of $0.61^{\circ} \mathrm{C}$ and a tight thermal control at the desired setpoint $\left(29.62+/-0.15^{\circ} \mathrm{C}\right)$. One of the test sessions shows a slightly higher overshoot, close to $0.91^{\circ} \mathrm{C}$, but this seems a particular event, and not a definite trend.

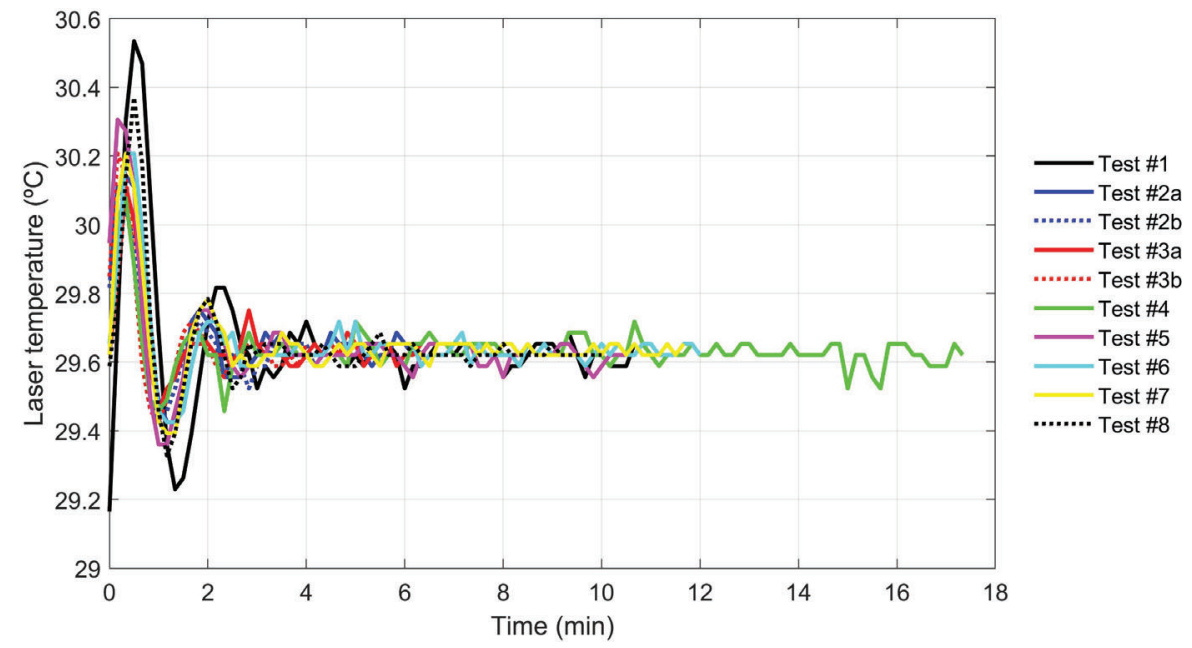

Figure 3. Laser temperature house-keeping during the complete laser on sequence. PID constants are system parameters that can be modified with telecommands, but they were kept constant during the AIV campaign after fixing them during TV testing at instrument level.

\subsection{Output power}

As for optical power, evaluated through the internal measurement in the feedback photodiode, the results show a performance consistent to the targeted output, between $20 \mathrm{~mW}$ and $30 \mathrm{~mW}$. At the selected temperature setpoint, the laser module was expected to work in constant current mode, providing almost $30 \mathrm{~mW}$ with an overall stability between $+/-5 \%$ and $+/-10 \%$. Most test sessions fulfill this requirement, but the tests $\# 2 \mathrm{a}$ and $\# 2 \mathrm{~b}$ fail to achieve this target after the initial temperature fluctuations. The behavior within this particular session is nevertheless coherent, with both consecutive measurements providing a similar outcome. We strongly believe that this result was due to the short testing time, since it is relevant to wait for internal stable thermal conditions before switching on the laser unit in order to improve performance repeatability. Often these instrument tests were not real performance tests, just short, quick functional tests planned together with other complex testing tasks, and they were used only to check that the instrument has not suffered any relevant damage or significant performance degradation. 


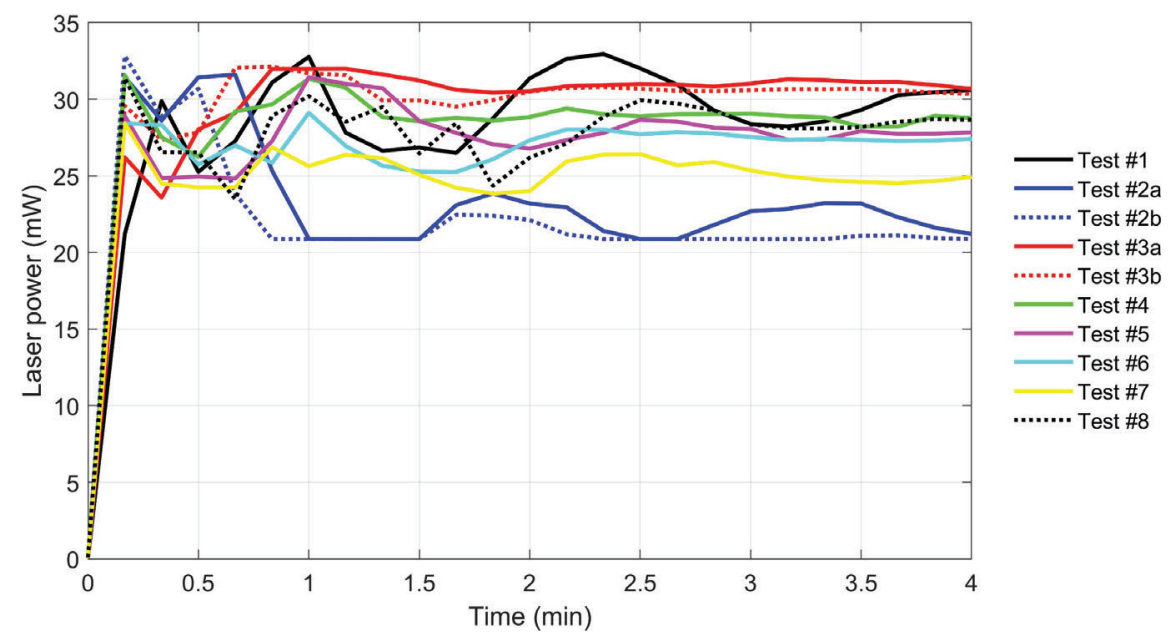

Figure 4. Estimated laser output power, obtained from internal monitoring photodiode, for the analyzed test sessions. Shortterm stability displayed in the figure is equivalent to the long-term stability expected in real sample measurements.

\subsection{Spectral performance}

Within the analyzed test session, laser spectral performance is good from a qualitative point of view. Side-mode suppression ratio, even if not perfect, does not affect seriously the quality of the Raman spectra obtained by the instrument. This can be seen in Figure 5, where the presence of a laser secondary peak can be visually assessed. In some of these spectra, the laser secondary peak appears indeed, indicating that the $>20 \mathrm{~dB}$ requirement (equivalent to less than $1 \%$ of the energy in the main peak) is not always fulfilled. Our estimates using the procedure described before give in fact values ranging from $12 \mathrm{~dB}$ to $8 \mathrm{~dB}$, but these estimates could be affected by several sources of noise, including Poisson noise from background signal (dark current and fluorescence) and the fitting of the Raman peak, that probably should follow a Lorentzian shape. In fact, we have found no real traces of the laser secondary peak in the best spectra. Even though not completely accurate, these spectral performance estimates are useful to track the unit performance.

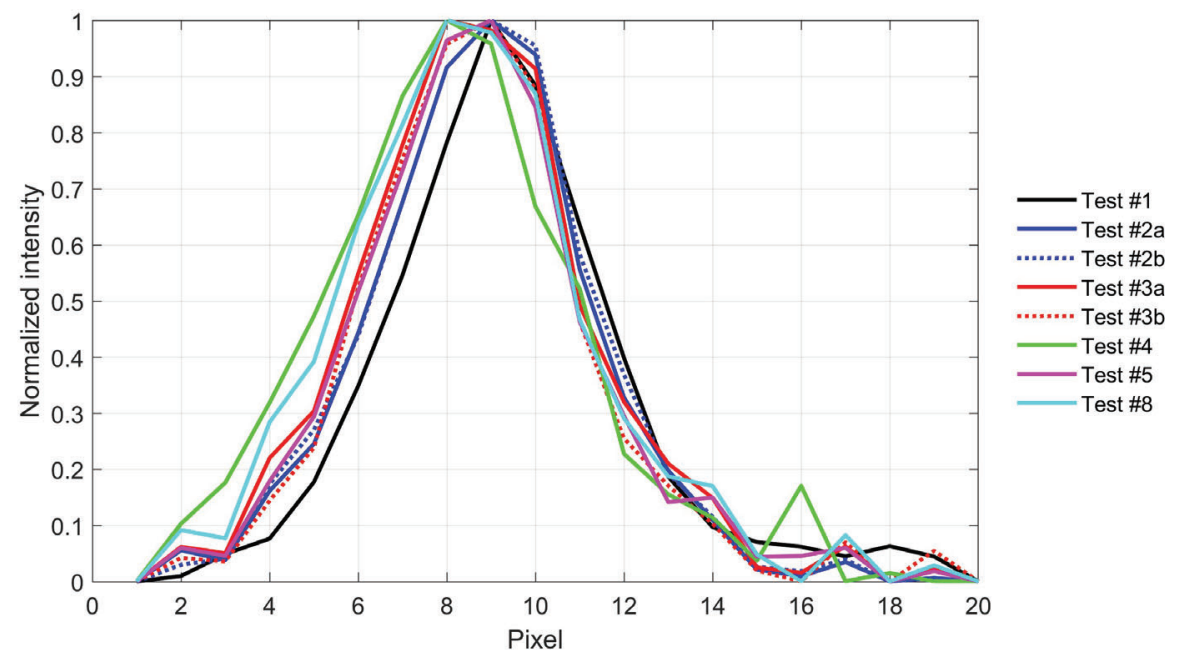

Figure 5. Raman peak from RLS calibration target Raman spectrum, with background and fluorescence removed and normalized, for all test sessions analyzed. Laser secondary peak creates a small hump around pixel 14, noticeable on test \#8. 


\section{CONCLUSIONS AND FUTURE WORK}

We have developed procedures aimed at assessing the RLS FM laser module performance once delivered to the next levels in the integration chain. These procedures are highly valuable for the definition of the laser performance assessment engineering tools to be used during standard scientific operation in Mars. They have demonstrated the need of refined analysis that could overcome the many limitations found when working with the instrument telemetries, with the spectral sampling of the instrument and with the noise sources associated to the instrument calibration target, such as fluorescence and the intrinsic Raman peaks linewidth. The more relevant output is, nevertheless, that the laser module maintains its expected performance, with just a minor shift in spectral performance, which is not optimal but good enough for scientific measurements. Since on-ground RLS FM testing will be quite limited from now on until the ExoMars rover launch date, we will keep enhancing these tools with the RLS instrument flight spare, that is already completely integrated and operative inside our cleanrooms at INTA.

\section{ACKNOWLEDGEMENTS}

This work has been funded by the Spanish MINECO (Ministerio de Economía y Competitividad) through ESP201456138-C3-1-R project.

\section{REFERENCES}

[1] Rull, F., Maurice, S., Hutchinson, I., Moral, A., Perez, C., Diaz, C., ... and Vago, J. L., “The Raman laser spectrometer for the ExoMars rover mission to Mars," Astrobiology 17(6-7), 627-654 (2017).

[2] Ribes-Pleguezuelo, P., Inza, A. M., Basset, M. G., Rodríguez, P., Rodríguez, G., Laudisio, M., ... and Tünnermann, A., "Assembly processes comparison for a miniaturized laser used for the Exomars European Space Agency mission,” Opt. Eng. 55(11), 116107 (2016).

[3] Benito-Parejo, M., Rodríguez-Pérez, P., Marín, Á., Rodríguez-Prieto, J. A., Canchal, R., Moral, A., and Rull, F., "Characterization Procedure of the Flight Laser Modules for the ExoMars Raman Laser Spectrometer," Proc. $8^{\text {th }}$ Int. Conf. Photonics, Opt. Laser Technol., 107-113 (2020).

[4] Lopez-Reyes, G., Saiz, J., Guzman, A., Moral, A., Perez, C., Rull, F., ... and Medina, J., "RLS FM performance characterization and calibration campaign with the Instrument Data Analysis Tool (IDAT)," Eur. Planet. Sci. Congr. EPSC2018-1132 (2018).

[5] Zafra, J., Quintana, C., Diez, M., Ibarmia, S., Seoane, L., Ramiro, M., ... and Prieto-Ballesteros, O., "RASTA: a supporting tool to manage all the data generated by the RLS instrument for ExoMars," 14 ${ }^{\text {th }}$ Europlanet Sci. Congr. EPSC2020-1086 (2020).

[6] Lopez-Reyes, G., Pilorget, C., Moral, A. G., Manrique, J. A., Sanz, A., Berrocal, A., ... and Vago, J. L., "Raman Laser Spectrometer (RLS) calibration target design to allow onboard combined science between the RLS and MicrOmega instruments on the ExoMars rover,' J. Raman Spectrosc. 51(9), 1718-1730 (2020). 